\title{
The role of curcumin in liver diseases
}

\author{
Antonio Riccardo Buonomo ${ }^{1}$, Riccardo Scotto ${ }^{1}$, Salvatore Nappa ${ }^{1}$, Michele Arcopinto ${ }^{2}$, \\ Andrea Salzano ${ }^{2,3}$, Alberto Maria Marra ${ }^{4}$, Roberta D’Assante', Emanuela Zappulo', \\ Guglielmo Borgia ${ }^{1}$, Ivan Gentile ${ }^{1}$
}

\begin{abstract}
${ }^{1}$ Department of Clinical Medicine and Surgery - Section of Infectious Diseases, University of Naples "Federico II", Naples, Italy

2Department of Translational Medical Science, University of Naples "Federico II", Naples, Italy

${ }^{3}$ Department of Cardiovascular Sciences and NIHR Leicester Biomedical Research Centre, University of Leicester, Glenfield Hospital, Groby Road, Leicester, UK ${ }^{4}$ IRCSS SDN, Naples, Italy
\end{abstract}

Submitted: 17 July 2017

Accepted: 20 October 2017

Arch Med Sci 2019; 15 (6): 1608-1620

DOI: https://doi.org/10.5114/aoms.2018.73596

Copyright $\odot 2018$ Termedia \& Banach

Liver diseases are among the most important health problems worldwide. In developed countries, overnutrition and alcohol abuse represent the major causes of liver disease, whereas infectious diseases - especially from viruses and parasites - are the main cause in Africa and Asia. Moreover, mortality rates from liver diseases are increasing rather than declining worldwide [1]. About $2 \%$ of the world's population is chronically infected with hepatitis C virus (HCV) [2], while the prevalence of hepatitis $B$ virus (HBV) in the global population is estimated to be $3.7 \%$ [3]: these viruses are the most common underlying agents in infection-related cirrhosis.

The availability of a new class of direct antivirals agents (DAA) against $\mathrm{HCV}$ is probably going to change the epidemiology of HCV infection. Results from randomized clinical trials (RCT) testing DAA showed a sustained viral response (SVR) rate up to $100 \%$ [2], thus making realizable the prospective of HCV global eradication [4]. However, little is known about the evolution of liver disease after viral eradication has been achieved; the complete regression of hepatic fibrosis is probably unachievable [5].

Liver fibrosis, and the subsequent cirrhosis, are the consequences of chronic liver injury due to several causes: viral cytotoxicity, oxidative stress, toxic stress (e.g. drugs, alcohol) and metabolic damage [6, 7].

Curcumin is the most important curcuminoid of turmeric, and it has been identified as the main factor responsible for its biological activities [8], including potent anti-oxidant and anti-inflammatory effects as well as the ability to modulate several signaling mechanisms [9-12]. Oxidative stress plays an important role in many chronic diseases and in carcinogenesis [13-15]. Several studies have shown that curcuminoid compounds can act as free-radical scavengers by reducing lipid peroxidation mediated by free radicals [16-18]. One of the most important discoveries in this field has been the curcumin-mediated suppression of nuclear factor $\kappa B(N F-\kappa B)$, which has a key role in triggering the inflammatory cascade in most chronic illnesses [19-21]. Importantly, NF- $\kappa B-d e p e n d e n t$ gene products also prevent apoptosis and promote cell proliferation, invasion and angiogenesis [22-26].

This review will focus on curcumin's role in preventing liver damage progression in different liver diseases, by interfering not only with in-

\author{
Corresponding author: \\ Dr. Riccardo Scotto \\ Department of Clinical \\ Medicine and Surgery \\ Section of Infectious Diseases \\ University of Naples \\ "Federico II" \\ Via Pansini, 5 \\ 80131 Naples, Italy \\ Phone: +393495601446 \\ E-mail: ri.scotto@gmail.com
}


flammation and fibrosis, i.e. in non-alcoholic fatty liver disease (NAFLD), but also with HCV replication and other mechanisms involved in hepatocarcinoma (HCC) development in HCV-related chronic liver disease.

\section{Curcumin bioavailability}

The interest in curcumin for chronic diseases has recently increased due to its anti-oxidant, antiinflammatory and anti-tumoral properties [27]. However, the pharmacodynamic actions of curcumin described in pre-clinical trials are limited by its unfavorable pharmacokinetic profile. In fact, curcumin showed low absorption due to its poor solubility in water and fast liver and intestinal metabolism, which contribute to its rapid excretion [28]. Moreover, it is highly unstable and rapidly hydrolyzed at physiological pH [29], and undergoes intense hepatic biotransformation (by conjugation and CYP reduction enzymes with prevalent excretion through the bile) [30].

In order to overcome curcumin's unfavorable pharmacokinetic profile, several formulations have been proposed, including curcumin-loaded nanoparticles [31], complexes with phospholipids [32], microemulsifying [33] and association with drug bioenhancers [34, 35]. Of particular interest is the delivery system of curcumin developed by Sasaki et al. [36] named Theracurmin, which consists of a highly absorptive formulation dispersed with colloidal nanoparticles. Oral administration of Theracurmin guaranteed a value of area under the curve (AUC) 40-fold higher compared with curcumin powder.

\section{Curcumin and liver damage}

\section{Inflammation and fibrosis}

Activation of hepatic stellate cells (HSCs) promotes liver fibrosis regardless of etiology. The mechanisms of HSC activation in vivo are often investigated with the fibrotic carbon tetrachloride $\left(\mathrm{CCl}_{4}\right)$ in animal models. In these models, curcumin showed a major role as an inhibitor of HSC activation; it also seems to be able to reduce liver damage, as well as the a-SMA and procollagen expression in the liver, when administered in $\mathrm{CCl}_{4}$-induced liver fibrosis models for 4-8 weeks. The recognized mechanism of action in those models included curcumin's ability to target multiple sites, such as platelet-derived growth factor-b receptor (PDGF-bR) [37], matrix metalloproteinases (MMPs) [37, 38], tissue growth factor b (TGFb) [39, 40], peroxisome proliferator-activated receptors (PPARc) [41], toll-like receptors (TLRs) [42], apoptotic pathway $[43,44]$, inflammatory cytokines [41, 42, 45, 46] and microRNAs [47, 48]. Recently, curcumin's capacity to increase cyclic adenosine monophosphate levels, which leads to an increase in the number of mitochondrial DNA duplicates, has been demonstrated [49].

A growing body of evidence has shown that leptin and its receptor (Ob-R) play an important role in activating HSCs and in the subsequent development of fibrosis. An in vitro study showed that curcumin is able to interrupt leptin signaling that would lead to HSC activation. It exerts this effect by inhibition of phosphorylation of Ob-R and suppression of $\mathrm{Ob}-\mathrm{R}$ gene expression by stimulating PPARc activity and reducing oxidative stress [50].

Moreover, curcumin appears to be effective in vitro in inhibiting hyperglycemia-induced HSC activation [51]. It has been shown that curcumin suppression of membrane translocation and gene expression of glucose transporter-2 leads to a reduction of intracellular glucose level of HSCs [51]. The same authors reported that curcumin inhibits the insulin receptor substrates (IRS)/PI3K/AKT signaling pathway, which links leptin with the insulin pathway, preventing the translocation of glucose transporter-4. It suppresses the PKA activity while increasing the activity of AMP-activated protein kinase (AMPK), thus increasing the glucokinase activity and subsequently the conversion of glucose to glucose-6-phosphate; the final effect is a reduction of glucose levels in HSCs [52].

Khan et al. [53] reported that curcumin also has anti-glycation properties: advanced glycation end-products (AGEs) are compounds formed by oxidative and non-oxidative reactions between proteins and/or lipids and carbohydrates [54] and their production is increased by hyperglycemia. Aging, chronic renal failure and diabetes facilitate the accumulation of AGEs in tissues and blood and lead to inflammation and liver fibrogenesis [55]. Amadori products are intermediates in the formation of AGEs; their conversion to AGEs is partially caused by oxidative reactions [56]; anti-oxidant agents may thus prevent the deposition of AGEs in tissues [57] which are involved in HSC activation. Several studies have shown the ability of curcumin to reduce HSC activation thanks to its anti-glycation properties [50, 58, 59]. However, there is no evidence for the role of curcumin in regulating gene expression of RAGE and AGE-R1 (which are the two categories of AGE receptors) in vivo. Lipid metabolism is implicated elsewhere in HSC activation, and curcumin appears to be also effective in inhibiting this pathway. In fact, in vitro studies have highlighted the effect of curcumin in increasing intracellular lipid accumulation in HSCS through the induction of lipogenesis-related genes, such as SREBP-1c, PPARc, and C/EBP a, leading to inhibition of HSC activation [60]. In addition, different studies have also shown significant improvement in the lipid metabolism and delay in hepatic 
fibrosis progression in rats and mice with steatohepatitis treated with curcumin [61-63].

As shown in in vitro models, another curcumin-mediated mechanism of inhibition of HSC activation is the elicitation of AMPK activity, which induces the expression of genes involved in lipid accumulation as well as in the increase of intracellular fatty acids and triglycerides levels [60]. In fact, the HSC activation is facilitated by reduction of lipid storage capacity and intracellular lipid droplets, as well as by suppression of PPARc, C/EBPa, SREBP 1 and other transcription factors $[64,65]$. In detail, hepatic AMPK leads to a reduction in glucose output with a subsequent lowering of blood glucose; this effect is exerted by high AMP and low ATP concentrations [66]. AMPK is also believed to regulate lipogenesis and SREBP 1 activation [67]. Moreover, as reported in studies conducted by Kang et al. [68-70], curcumin exerts several effects that also reduce HSC activation: it suppresses the effects of specific protein-1, which is involved in attenuating the expression of SREBP 2, it reduces the gene expression of lectin-like oxidized LDL receptor-1 (LOX-1) and it suppresses the expression of LDL receptor.

Curcumin also has a role in other steps of fibrogenesis when increased extracellular proteins are deposited in the extracellular space of the liver. This deposition is regulated by the action of a group of enzymes called MMPs and their specific inhibitors, tissue inhibitors of metalloproteinases (TIMPS). When this balance is broken by hyperactivation of HSCs, fibrosis occurs. Available evidence showed that curcumin plays a role in downregulation of TIMP-1 and TIMP-2 in vivo [38, 71-73] and in vitro [74], while it upregulates MMP-2 [73], MMP-7 [72], MMP-9 [73] and MMP-13 [38, 72]. This leads to inhibition of HSC activation due to the degradation of one of the main components of the ECM, the fibrillar collagens. Furthermore, another crucial mechanism involved in liver fibrosis is the reduction of PPARc expression in HSCs: in fact, PPARc is highly expressed in inactive HSCs, while its activity is reduced when HSCs are activated both in vivo and in vitro $[65,75,76]$. Curcumin may thus interfere with liver fibrosis due to its role in induction of the expression of PPAR-c, which results in a reduction of vitamin $A$ and lipid-droplet storing capability, deposition of ECM in the extracellular space and expression of a-SMA and type I collagen- $\alpha 1$, as well as in elicitation of cell proliferation and growth. Finally, the curcumin-mediated PPARc activation results in inhibition of trans-activating activity of NF- $\mathrm{BB}$, while - when a specific PPARc antagonist is used - higher HSC proliferation is observed.

Considering that adipokines, such as leptin and adiponectin, have an important role in regulation adipocyte energy metabolism [77, 78], curcumin has anti-diabetic effects through the suppression of NF- $\kappa B$ in adipocytes and the reduction

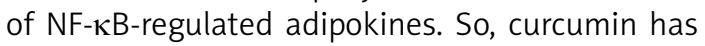
anti-diabetic effects that could be considered as valuable targets for diabetes mellitus [79].

With regard to clinical trials in humans, a randomized, double-blind, placebo-controlled trial [80] showed that a 12-week fermented turmeric powder (FTP) treatment was associated with a statistically significant decline in both ALT and AST levels in 30 subjects with mild elevated transaminases concentrations at baseline (with the possible speculation of a reduction of the hepatic cell damage by FTP therapy). One limitation of this study was the unknown etiology of the transaminase elevation in the enrolled patients. However, patients with chronic HCV and HBV infections, as well as those with abnormal transferrin saturation, were excluded before randomization (Table I).

\section{Non-alcoholic fatty liver disease}

Non-alcoholic fatty liver disease (NAFLD) is the most prevalent chronic liver disease, and it is associated with the classic clinical components of the metabolic syndrome: obesity, diabetes, dyslipidemia, insulin resistance [81]. These predisposing conditions are highly prevalent in the western countries; hence NAFLD has become a public health concern with an estimated prevalence of $6-35 \%$ among adults worldwide [82]. Liver steatosis is recognized as a decisive "first hit" in the pathogenesis of the disease and it is defined by the presence of cytoplasmic triglyceride (TG) droplets in > 5\% of hepatocytes in the absence of significant alcohol consumption [83]. Therefore, modifications in dietary and lifestyle habits represent the first therapeutic target to prevent NAFLD or its progression to non-alcoholic steatohepatitis (NASH)/cirrhosis [84]. However, as in other conditions requiring considerable changes in dietary habits, most patients fail to comply with effective lifestyle modifications. The NAFLD is demonstrated to evolve in severe liver fibrosis and cirrhosis. To date, there is no specific drug available on the market able to effectively reverse liver steatosis or slow NAFLD progression. Therefore, there is a growing interest to find effective and safe treatments for this important clinical problem. Natural products have been the subject of increasing attention owing to their multi-target action and overall safety in several conditions [85-88]. Due to the possible protective action that curcumin exerts on the liver, and the beneficial effect shown on the associated conditions of NAFLD, including obesity [89], dyslipidemia [90], and impaired fasting glucose [91], this compound has been considered as a potential therapeutic 


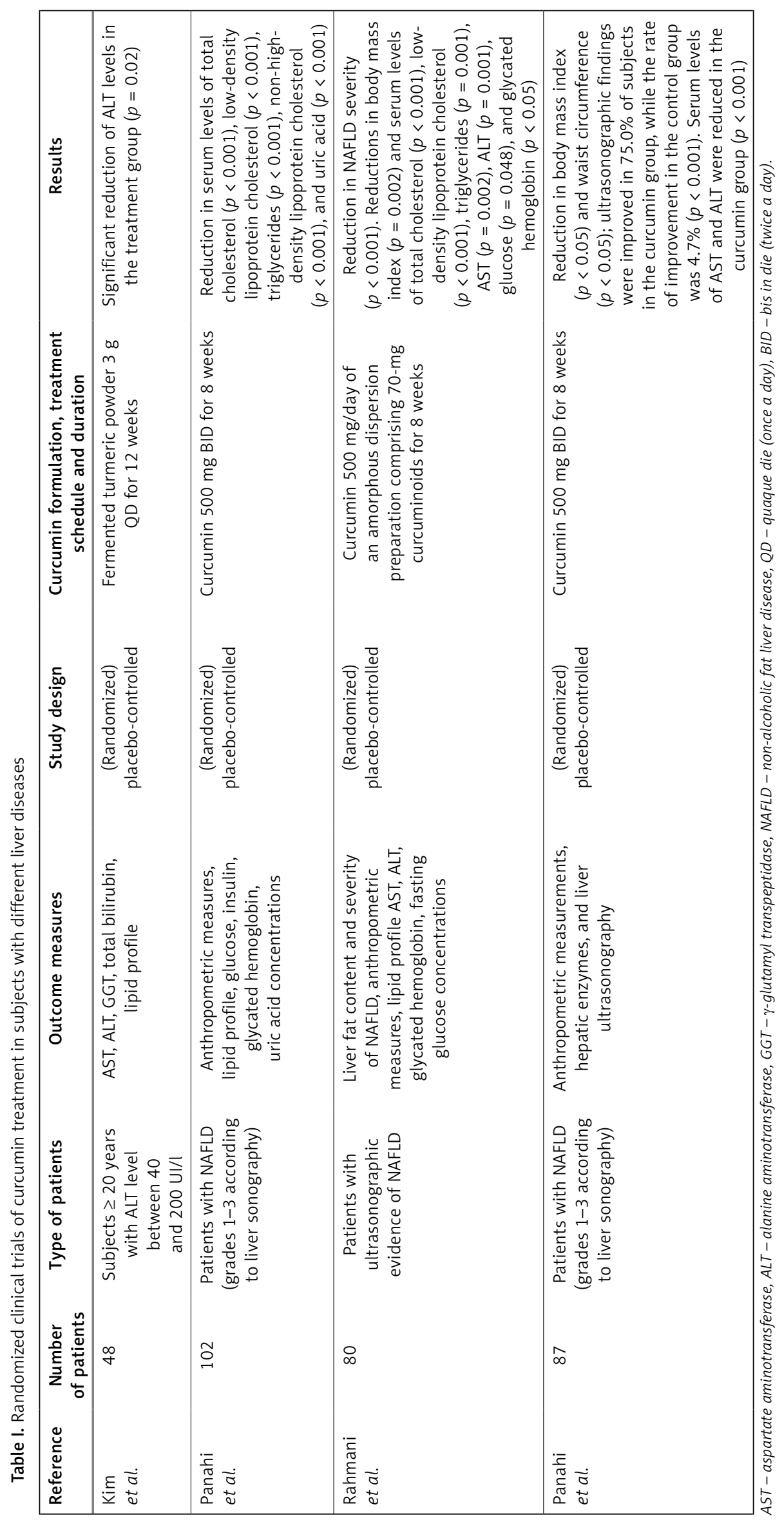


agent in NAFLD. In recent years, curcumin (and its derivates) has been tested in animal models of NAFLD with results elucidating possible mechanisms of action and paving the way to possible clinical use. In a methionine choline deficient (MCD)-diet-induced NAFLD mouse model, bisdemethoxycurcumin (BDMC), a close curcumin analogue, was investigated for its potential hepatoprotective effect. To this aim, a control group (normal diet), a NAFLD group (MCD diet), and a MCD + BDMC group were studied after a 4-week treatment. Convincing results showed that BDMC was associated with significantly lower circulating aspartate aminotransferase (AST), alanine aminotransferase (ALT) and inflammatory markers (IL-6 and TNF- $\alpha$ ) compared to MCD mice. Moreover, TG levels, expressed as $\mathrm{mg} /$ total liver tissue, were very similar to the ones measured in the control group. In this study, the BDMC also showed an inhibitory effect on liver lipogenesis by reducing the expression of genes related to fatty acid synthesis, which are generally found upregulated in NAFLD. In a subsequent study, male Sprague-Dawley rats were fed with a high-fat and high-sugar diet for 8 weeks (NAFLD group), then randomly assigned to curcumin by oral gavage $(80 \mathrm{mg} / \mathrm{kg} /$ day $)$ or an equal volume of cellulose and compared with a control group (normal diet). Biohumoral markers, including ALT, TG, total cholesterol (TC) and HOMA index, as well as liver histology parameters, were consistent with a significant recovery of the clinical features of the NAFLD in curcumin-fed rats. In this study, a specific pathophysiologic effect has been proposed: by measuring adiponectin precursor (ADIPOQ) mRNA, protein expressions and DNA methylation status and by analyzing the correlations between these and changes in TC, TG, ALT and HOMA-I, DNA methylation of ADIPOQ was found to be one of the mechanisms by which curcumin executes its hepatoprotective function in NAFLD [92]. This first study testing curcumin in humans with NAFLD (grades 1-3 in ascending severity, according to liver ultrasonography definition) evaluated the metabolic effects in subjects randomly assigned to a curcumin $(1000 \mathrm{mg} /$ day in 2 divided doses) $(n=44)$ or control $(n=43)$ group for a period of 8 weeks. Serum levels of total cholesterol, low-density lipoprotein cholesterol, triglycerides, non-high-density lipoprotein cholesterol and also uric acid significantly decreased in the curcumin group [93]. The highest quality trial testing curcumin compounds in subjects with NAFLD was published in 2016 by Rahmani et al. [94] and added a placebo-controlled design and an ultrasound end-point. In this study, 80 subjects of both sexes, selected as in the study presented above, were $1: 1$ randomly assigned to curcumin $(500 \mathrm{mg} /$ day of an amorphous dispersion preparation comprising 70-mg curcuminoids) or placebo supple- mentation for 8 weeks. Most anthropometric and biohumoral parameters (total cholesterol, LDL-C, $\mathrm{HDL}-\mathrm{C}$, triglycerides, BMI, body weight, ALT, AST, $\mathrm{HbA}_{1 \mathrm{c}}$ ) were found significantly improved after the supplementation in the curcumin group compared with placebo. Similarly, a comparison of liver ultrasonographic findings (severity of liver steatosis on a scale from 1 to 3) at baseline and after 8 weeks revealed a significant improvement in almost $80 \%$ of subjects in the curcumin versus $27 \%$ in the placebo group. Interestingly, the effect of curcumin in reducing US evidence of liver fat remained significant after adjustment for possible confounding factors, including baseline levels of TG, $\mathrm{HbA}_{1 \mathrm{c}}, \mathrm{ALT}$ and changes in body weight.

Although direct comparison between the two human studies available in the literature are limited by the different curcumin posology employed, there are striking similarities in the overall beneficial effect on the metabolic profile in treated subjects. Findings of this randomized controlled trial are also summarized in Table I.

Moreover, a more recent randomized placebo-control trial of 87 subjects confirmed these data [95]. After 8 weeks of curcumin (phytosomal form, at daily dose of $1 \mathrm{~g}$ ), a reduction in body mass index and waist circumference and an improvement in ultrasonographic liver findings were observed, with a significant reduction of ALT and AST.

In a previous randomized double-blind placebo-control trial of the same group, 117 subjects with metabolic syndrome were randomly assigned to curcuminoids or placebo [96]. After 8 weeks of follow up, supplementation with curcuminoid (at a daily dose of $1 \mathrm{~g}$ ) and piperine (at a daily dose of $10 \mathrm{mg}$ ) combination significantly improved the oxidative and inflammatory status of patients. In particular, there was observed an improvement in serum superoxide dismutase activities and a reduction in concentration malondialdehyde and C-reactive protein. These results are in line with previous studies [97].

Of note, the important role of curcumin in a clinical setting was recently reviewed by an International Lipid Panel expert [98]. In this work, the authors concluded that an evidence-based approach to the use of lipid-lowering nutraceuticals could improve the quality of the treatment, including therapy adherence, and achievement of the LDL-C goal in clinical practice, an important endpoint in cardiovascular disease [99-102]. Moreover, in Table II, ongoing or future trials registered at clinicaltrials.gov to be performed with curcumin in metabolic and hepatic diseases are listed.

\section{Curcumin and HCV replication}

Kim et al. demonstrated that the induction of heme-oxygenase $1(\mathrm{HO}-1)$ and the inhibition of 


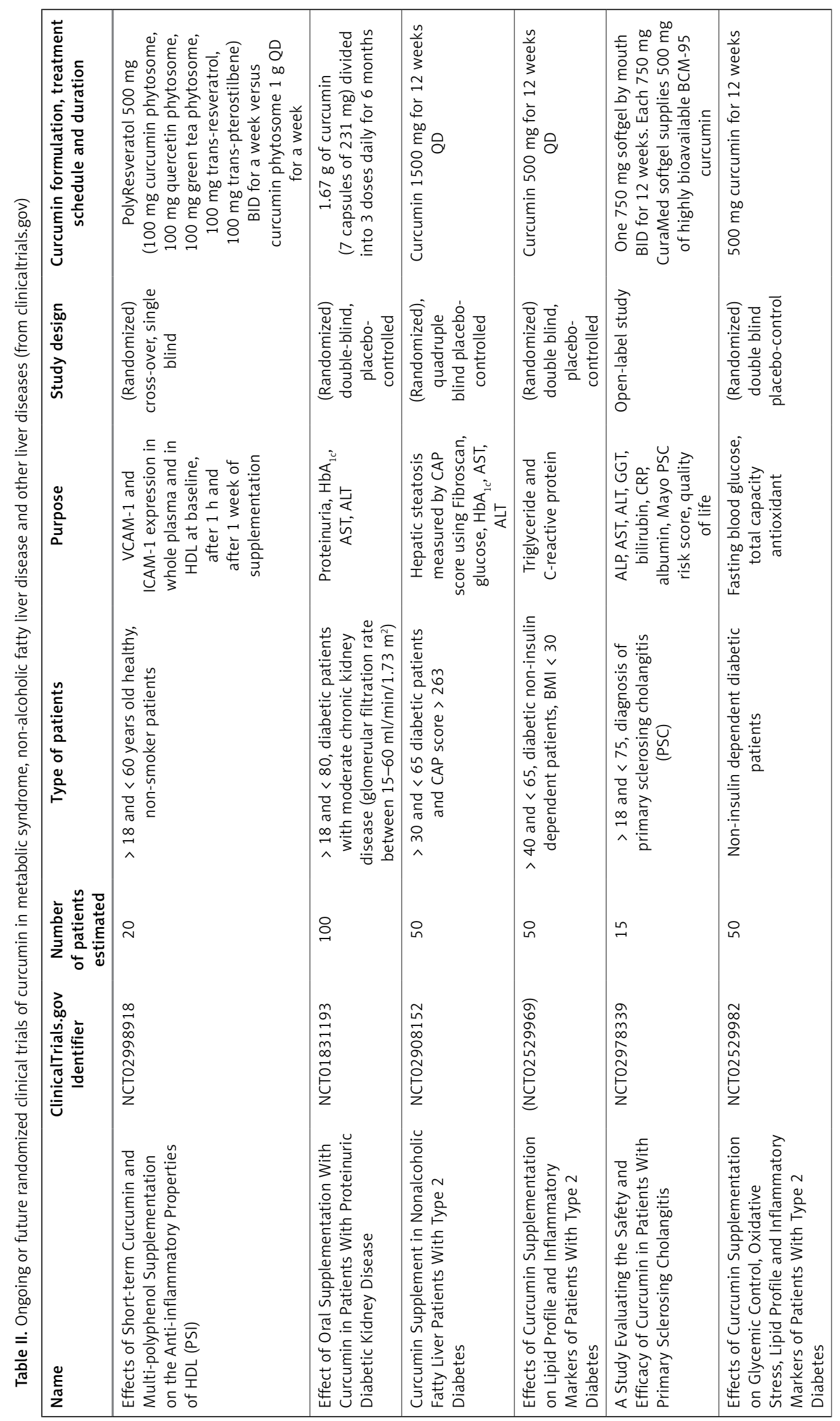




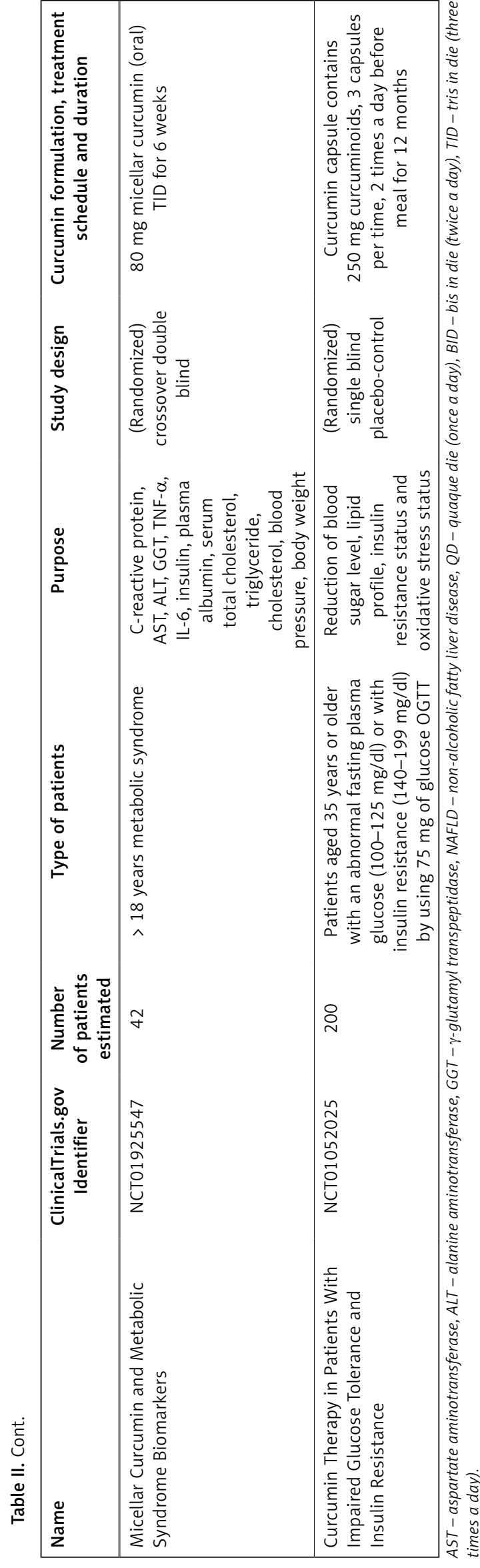

PI3K-AKT partially promote HCV replication. HO-1 is a curcumin-induced gene and it is a potential therapeutic protein for the regulation of homeostasis in several diseases [103]. Furthermore, HO-1 inhibits the replication of HCV [104]. In fact, its knock-down partially reverses the curcumin-mediated inhibition of $\mathrm{HCV}$ replication and since HO- 1 is induced by ROS, HO-1 and ROS may have a role in inhibiting HCV replication $[105,106]$. Kim et al. also showed that curcumin may have a role in the suppression of HCV replication by blocking the AKT-SREBP-1 pathway [107]. A recent study showed different mechanisms in curcumin-mediated HCV replication inhibition. First of all, they demonstrated that the main metabolized form of curcumin (THC), derived from the lack of $\alpha 1 \beta$ unsaturated ketone groups, is not active in inhibiting HCV replication. Therefore, they postulated that the key structures involved in this HCV inhibition process were the ketone groups; moreover, they discovered that curcumin was able to affect the entry pathway and had no activity against replication or assembly/release of infectious particles. Curcumin seemed to exert its activity directly impairing the ability of the virus to enter the target cells, and the proposed mechanism of action was a modulation of the fluidity of the viral membrane with no effect on the integrity of viral particles. Moreover, curcumin seems to be able to interfere with the cell-to-cell HCV spreading mechanism, and this capability may be correlated with its hydrophobicity by which curcumin could insert among lipids of the viral envelope, thereby increasing its rigidity and thus inhibiting its fusion capabilities.

\section{Curcumin and hepatocellular carcinoma (HCC)}

One of the earliest papers presenting evidence about the role of curcumin in prevention of HCC was published by Soni et al. in 1997 [108]. They showed that turmeric and curcumin inhibit the aflatoxin B1-induced mutagenesis in hepatic murine cells, reducing the genesis of gammaglutamyl-transpeptidase-positive foci. Furthermore, in 1998, Lin et al. [109] showed that curcumin decreases migration and invasion of a highly aggressive human HCC cell line through dose-dependent inhibition of MMP-9 secretion. In a N-diethylnitrosamine (DEN)-induced model of hepatocarcinogenesis conducted in $\mathrm{C} 3 \mathrm{H} / \mathrm{NeH}$ rats, Chuang et al. [110] found that administration of curcumin significantly decreases both incidence and multiplicity of HCC; similar results were achieved in subsequent studies conducted by different authors in female Wistar rats [111-113]: they all showed the protective role of curcumin in in vivo models of DEN-induced HCC. In these studies the suppressive effects of curcumin in the DENA-initiated al- 
teration of hepatic foci in rats was accompanied by the restoration of normal levels of several hepatic metabolic enzymes. Busquets et al. [114] evaluated the effects of curcumin in a xenograft model of hepatocarcinogenesis; they demonstrated that the administration of curcumin can decrease the growth rate of HCC in Yoshida ascites hepatoma in rats, even with no influence on the tumor volume reduction, thus excluding a possible effect of curcumin on cell apoptosis. The effects of curcumin in combination with standard chemotherapeutic agents have also been investigated in vitro. In an interesting study, Notarbartolo et al. [115] showed that the combination of curcumin with cisplatin and doxorubicin results in synergistic cell growth inhibition in HA22T/VGH cells. Despite the reported positive evidence in some preclinical models, several studies have documented the failure of curcumin in protecting against chemical hepatocarcinogenesis in rodents. Curcumin failed to prevent HCC in rats treated with different chemical tumor inducers including $N$-ethyl- $N$-hydroxyethylnitrosamine, 3, 2'-methyl-4-aminobiphenyl [116] 3, 2'-methyl-4-aminobiphenyl, DENA/2-amino-3,8-dimethylimidazo[4,5-f] quinoxaline [117, 118], and 3,3',4,4'-tetrachlo-robiphenyl [119]. Curcumin also failed to protect against copper-induced HCC in rats [120]. In the aforementioned studies, curcumin was administered in a relatively low dose, which probably prevented the occur- rence of its pro-oxidants effects. In a very recent in vitro study, Cao et al. [121] evaluated the concomitant administration of sorafenib and curcumin; the authors assembled sorafenib and curcumin codelivered nanoparticles (SCN) and then measured their antiproliferative effects, in comparison with free sorafenib, curcumin and a physical mixture of sorafenib and curcumin (Sora + Cur). The coassembled SCN presented significantly enhanced antiproliferative effects compared to sorafenib, curcumin (6.0 and 1.5-fold higher reduction of tumor growth rate, respectively), and their physical mixture. In the same study, in vivo antiproliferative and antiangiogenic activities of SCN were determined by immunohistochemistry measurements. The captured images showed that the neovasculature was detected in the tumor sections from control, sorafenib, curcumin, and Sora + Cur groups, but was barely detected in the SCN-treated group, which implicated the higher antiangiogenic capability of SCN over other chemotherapy regimens. This evidence verified the enhanced therapeutic efficacy on in vivo antiproliferative and antiangiogenic activities by SCN.

\section{Conclusions}

There are numerous etiological factors able to elicit chronic liver inflammation that could lead to hepatic fibrosis and cirrhosis, including metabol-

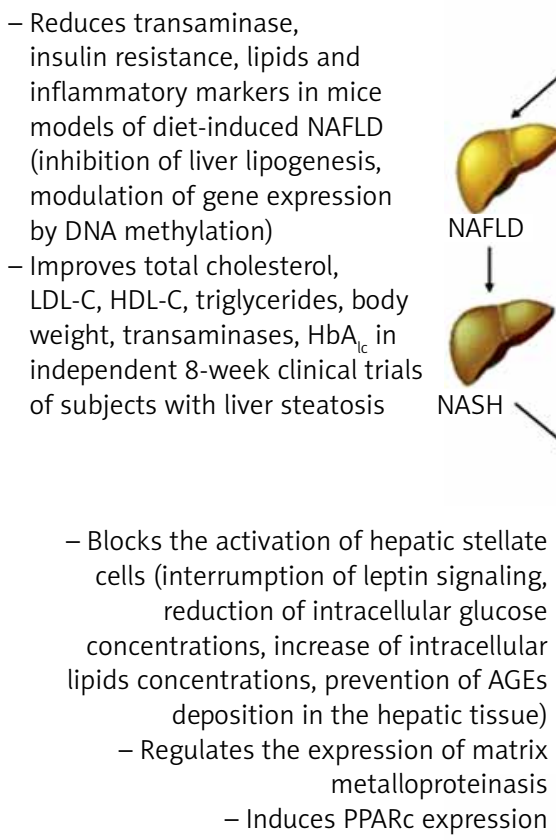

- Blocks the activation of hepatic stellate cells (interrumption of leptin signaling, reduction of intracellular glucose concentrations, increase of intracellular lipids concentrations, prevention of AGEs deposition in the hepatic tissue)

- Regulates the expression of matrix metalloproteinasis

- Induces PPARc expression
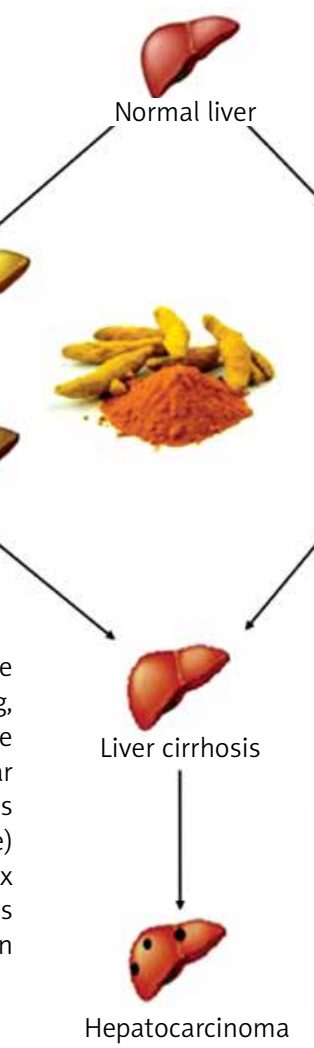

- Inhibits HCV replication by induction of HO-1 gene and inhibition of AKT-SREBP-1 pathway

- Impairs the ability of the virus to enter the target cells (modulation of the fluidity of the viral membrane)
- Decreases migration and invasion of human $\mathrm{HCC}$ cell line throughout inhibition of matrix metalloproteinase-9

- Pro-apoptotic and anti-proliferative effects in HCC (ROS induction, attenuation of telomerases activity, downregulation of NOTCH-I pathway)

Figure 1. Curcumin main effects on sequential stages of liver pathology 
ic, infectious and autoimmune diseases. Despite the availability of etiologic-specific treatments for several liver diseases, no drugs or medications are currently approved for the regression of hepatic fibrosis and cirrhosis, which represent the final consequences of chronic liver injury. The interest in anti-fibrotic therapeutic or preventive agents has recently risen due to the availability of a new class of DAA against HCV. Whereas the clinical efficacy of these brand new medications has been widely demonstrated in eradicating HCV infection [2, 122-128], little is known about their effects on liver fibrosis and a significant histological improvement of cirrhosis with antiviral agents is probably unfeasible.

Curcumin is a natural compound easily extractable from turmeric and is widely used in middle-eastern diets. Several studies have uncovered its role in the modulation of many biological mechanisms involved in liver injury, and they are summarized in Figure 1.

The available data make curcumin a promising phytotherapy in chronic hepatitis and a potential therapeutic agent for regression of liver fibrosis and cirrhosis, especially in the setting of HCV infection, which could be easily eradicated with many direct-acting antivirals. Even though results from studies conducted so far are encouraging, several critical issues still must be overcome before curcumin may become available in clinical practice. The above-mentioned poor bioavailability of curcumin is a critical limiting factor which affects the drug pharmacodynamics, and then the use of a novel drug delivery system for curcumin must be considered when testing its clinical effects.

\section{Conflict of interest}

The authors declare no conflict of interest.

\section{References}

1. Williams R. Global challenges in liver disease. Hepatology 2006; 44: 521-6.

2. Gentile I, Buonomo AR, Zappulo E, Borgia G. Interferon-free therapies for chronic hepatitis C: toward a hepatitis $C$ virus-free world? Expert Rev Anti Infect Ther 2014; 12: 763-73.

3. Locarnini S, Hatzakis A, Chen DS, Lok A. Strategies to control hepatitis B: public policy, epidemiology, vaccine and drugs. J Hepatol 2015; 62 (1 Suppl): S76-86.

4. Gentile I, Maraolo AE, Niola M, Graziano V, Borgia G, Paternoster $M$. Limiting the access to direct-acting antivirals against HCV: an ethical dilemma. Expert Rev Gastroenterol Hepatol 2016; 10: 1227-34.

5. Knop V, Hoppe D, Welzel T, et al. Regression of fibrosis and portal hypertension in HCV-associated cirrhosis and sustained virologic response after interferon-free antiviral therapy. J Viral Hepat 2016; 23: 994-1002.

6. Zhou WC, Zhang QB, Qiao L. Pathogenesis of liver cirrhosis. World J Gastroenterol 2014; 20: 7312-24.
7. Martínez-Esparza M, Tristán-Manzano M, Ruiz-Alcaraz AJ, García-Peñarrubia P. Inflammatory status in human hepatic cirrhosis. World I Gastroenterol 2015; 21 : 11522-41.

8. Aggarwal BB, Sundaram C, Malani N, Ichikawa H. Curcumin: the Indian solid gold. Adv Exp Med Biol 2007; 595: 1-75.

9. Sethi G, Sung G, Aggarwal B. The role of curcumin in modern medicine. In: Herbal Drugs: Ethnomedicine to Modern Medicine. Ramawat KG (ed.). Springler-Verlag Berlin, Heidelberg 2009; 97-113.

10. Calabrese V, Bates TE, Mancuso C, et al. Curcumin and the cellular stress response in free radical-related diseases. Mol Nutr Food Res 2008; 52: 1062-73.

11. Kunnumakkara AB, Anand P, Aggarwal BB. Curcumin inhibits proliferation, invasion, angiogenesis and metastasis of different cancers through interaction with multiple cell signaling proteins. Cancer Lett 2008; 269: 199-225.

12. Sarkar FH, Li Y, Wang Z, Padhye S. Lesson learned from nature for the development of novel anti-cancer agents: implication of isoflavone, curcumin, and their synthetic analogs. Curr Pharm Des 2010; 16: 1801-12.

13. Bishayee A, Darvesh A. Oxidative stress in cancer and neurodegenerative diseases: prevention and treatment by dietary anti-oxidants. In: Free Radicals: Formation, Types and Effects. Kozyrev D, Slutsky V (eds). Nova Science Publishers, Inc Hauppage, NY 2010; 1-55.

14. Valko M, Rhodes CJ, Moncol J, Izakovic M, Mazur M. Free radicals, metals and antioxidants in oxidative stress-induced cancer. Chem Biol Interact 2006; 160: 1-40.

15. Chen G, Li X, Yang J, et al. Prognostic significance of cyclooxygenase-2 expression in patients with hepatocellular carcinoma: a meta-analysis. Arch Med Sci 2016; 12: 1110-7.

16. Sharma OP. Antioxidant activity of curcumin and related compounds. Biochem Pharmacol 1976; 25: 1811-2.

17. Osawa T, Sugiyama Y, Inayoshi M, Kawakishi S. Antioxidative activity of tetrahydrocurcuminoids. Biosci Biotechnol Biochem 2014; 59: 1609-12.

18. Ak T, Gülçin I. Antioxidant and radical scavenging properties of curcumin. Chem Biol Interact 2008; 174: 27-37.

19. Libby P. Inflammatory mechanisms: the molecular basis of inflammation and disease. Nutr Rev 2007; 65: S140-6.

20. Sethi G, Sung B, Aggarwal BB. Nuclear factor-kappaB activation: from bench to bedside. Exp Biol Med 2008; 233: 21-31.

21. Ralhan R, Pandey MK, Aggarwal BB. Nuclear factor-kappa $B$ links carcinogenic and chemopreventive agents. Front Biosci 2009; 1: 45-60.

22. Shishodia S, Amin HM, Lai R, Aggarwal BB. Curcumin (diferuloylmethane) inhibits constitutive NF-kappaB activation, induces $\mathrm{G} 1 / \mathrm{S}$ arrest, suppresses proliferation, and induces apoptosis in mantle cell lymphoma. Biochem Pharmacol 2005; 70: 700-13.

23. Aggarwal BB, Shishodia S, Takada Y, et al. Curcumin suppresses the paclitaxel-induced nuclear factor-kappaB pathway in breast cancer cells and inhibits lung metastasis of human breast cancer in nude mice. Clin Cancer Res 2005; 11: 7490-8.

24. Aggarwal S, Ichikawa H, Takada Y, Sandur SK, Shishodia S, Aggarwal BB. Curcumin (diferuloylmethane) down-regulates expression of cell proliferation and antiapoptotic and metastatic gene products through suppression of IkappaBalpha kinase and Akt activation. Mol Pharmacol 2006; 69: 195-206.

25. Kamat AM, Sethi G, Aggarwal BB. Curcumin potentiates the apoptotic effects of chemotherapeutic agents 
and cytokines through down-regulation of nuclear factor-kappaB and nuclear factor-kappaB-regulated gene products in IFN-alpha-sensitive and IFN-alpha-resistant human bladder cancer cell. Mol Cancer Ther 2007; 6: 1022-30.

26. Deeb D, Jiang H, Gao X, et al. Curcumin [1,7-bis(4-hydroxy-3-methoxyphenyl)-1-6-heptadine-3,5-dione; $\mathrm{C} 21 \mathrm{H} 2006$ sensitizes human prostate cancer cells to tumor necrosis factor-related apoptosis-inducing ligand/ Apo2L-induced apoptosis by suppressing nuclear factor-kappaB via inhibition of the prosurvival Akt signaling pathway. J Pharmacol Exp Ther 2007; 321: 616-25.

27. Gupta SC, Patchva S, Koh W, Aggarwal BB. Discovery of curcumin, a component of the golden spice, and its miraculous biological activities. Clin Exp Pharmacol Physiol 2012; 39: 283-99.

28. Anand P, Thomas SG, Kunnumakkara AB, et al. Biological activities of curcumin and its analogues (Congeners) made by man and Mother Nature. Biochem Pharmacol 2008; 76: 1590-611.

29. Mehanny M, Hathout RM, Geneidi AS, Mansour S. Exploring the use of nanocarrier systems to deliver the magical molecule; curcumin and its derivatives. J Control Release 2016; 225: 1-30.

30. Pan MH, Huang TM, Lin JK. Biotransformation of curcumin through reduction and glucuronidation in mice. Drug Metab Dispos 1999; 27: 486-94.

31. Gao Y, Li Z, Sun M, et al. Preparation, characterization, pharmacokinetics, and tissue distribution of curcumin nanosuspension with TPGS as stabilizer. Drug Dev Ind Pharm 2010; 36: 1225-34.

32. Thangapazham RL, Puri A, Tele S, Blumenthal R, Maheshwari RK. Evaluation of a nanotechnology-based carrier for delivery of curcumin in prostate cancer cells. Int J Oncol 2008; 32: 1119-23.

33. Cui J, Yu B, Zhao Y, et al. Enhancement of oral absorption of curcumin by self-microemulsifying drug delivery systems. Int J Pharm 2009; 371: 148-55.

34. Shoba G, Joy D, Joseph T, Majeed M, Rajendran R, Srinivas PS. Influence of piperine on the pharmacokinetics of curcumin in animals and human volunteers. Planta Med 1998; 64: 353-6.

35. Prasad S, Tyagi AK, Aggarwal BB. Recent developments in delivery, bioavailability, absorption and metabolism of curcumin: the golden pigment from golden spice. Cancer Res Treat 2014; 46: 2-18.

36. Sasaki H, Sunagawa Y, Takahashi K, et al. Innovative preparation of curcumin for improved oral bioavailability. Biol Pharm Bull 2011; 34: 660-5.

37. Zhang XP, Zhang F, Zhang ZL, et al. Acupuncture combined with curcumin disrupts platelet-derived growth factor beta receptor/extracellular signal-regulated kinase signalling and stimulates extracellular matrix degradation in carbon tetrachloride-induced hepatic fibrosis in rats. Acupunct Med 2012; 30: 324-30.

38. Morsy M, Abdalla A, Mahmoud A, Abdelwahab S, Mahmoud ME. Protective effects of curcumin, alpha-lipoic acid, and $\mathrm{N}$-acetylcysteine against carbon tetrachloride-induced liver fibrosis in rats. J Physiol Biochem 2012; 68: 29-35.

39. Yao Q, Xu B, Wang J, Liu H, Zhang S, Tu C. Inhibition by curcumin of multiple sites of the transforming growth factor-beta1 signalling pathway ameliorates the progression of liver fibrosis induced by carbon tetrachloride in rats. BMC Complement Altern Med 2012; 12: 156.

40. Reyes-Gordillo K, Segovia J, Shibayama M, et al. Curcumin prevents and reverses cirrhosis induced by bile duct obstruction or $\mathrm{CCl} 4$ in rats: role of TGF-beta modulation and oxidative stress. Fundam Clin Pharmacol 2008; 22: 417-27.

41. Fu Y, Zheng S, Lin J, Ryerse J, Chen A. Curcumin protects the rat liver from $\mathrm{CCl} 4$-caused injury and fibrogenesis by attenuating oxidative stress and suppressing inflammation. Mol Pharmacol 2008; 73: 399-409.

42. Tu C, Yao Q, Xu B, Wang J, Zhou C, Zhang S. Protective effects of curcumin against hepatic fibrosis induced by carbon tetrachloride: modulation of high-mobility group box 1, Toll-like receptor 4 and 2 expression. Food Chem Toxicol 2012; 50: 3343-51.

43. Shu JC, He YJ, Lv X, Ye GR, Wang LX. Curcumin prevents liver fibrosis by inducing apoptosis and suppressing activation of hepatic stellate cells. J Nat Med 2009; 63: 415-20.

44. Priya S, Sudhakaran PR. Curcumin-induced recovery from hepatic injury involves induction of apoptosis of activated hepatic stellate cells. Indian J Biochem Biophys 2008; 45: 317-25.

45. Bassiouny AR, Zaky A, Kandeel KM. Alteration of AP-endonuclease 1 expression in curcumin-treated fibrotic rats. Ann Hepatol 2011; 10: 516-30.

46. Wu SJ, Tam KW, Tsai YH, Chang CC, Chao JC. Curcumin and saikosaponin a inhibit chemical-induced liver inflammation and fibrosis in rats. Am J Chin Med 2010; 38: 99-111.

47. Hassan ZK, Al-Olayan EM. Curcumin reorganizes miRNA expression in a mouse model of liver fibrosis. Asian Pac J Cancer Prev 2012; 13: 5405-8.

48. Momtazi AA, Derosa G, Maffioli P, Banach M, Sahebkar A. Role of microRNAs in the therapeutic effects of curcumin in non-cancer diseases. Mol Diagn Ther 2016; 20: 335-45.

49. Sahebkar A, Saboni N, Pirro M, Banach M. Curcumin: an effective adjunct in patients with statin-associated muscle symptoms? J Cachexia Sarcopenia Muscle 2017; 8: 19-24.

50. Tang Y, Zheng S, Chen A. Curcumin eliminates leptin's effects on hepatic stellate cell activation via interrupting leptin signaling. Endocrinology 2009; 150: 3011-20.

51. Lin J, Chen A. Curcumin diminishes the impacts of hyperglycemia on the activation of hepatic stellate cells by suppressing membrane translocation and gene expression of glucose transporter-2. Mol Cell Endocrinol 2011; 333: 160-71.

52. Tang Y, Chen A. Curcumin prevents leptin raising glucose levels in hepatic stellate cells by blocking translocation of glucose transporter- 4 and increasing glucokinase. Br J Pharmacol 2010; 161: 1137-49.

53. Khan I, Ahmad H, Ahmad B. Anti-glycation and anti-oxidation properties of Capsicum frutescens and Curcuma longa fruits: possible role in prevention of diabetic complication. Pak J Pharm Sci 2014; 27: 1359-62.

54. Bierhaus A, Humpert PM, Morcos M, et al. Understanding RAGE, the receptor for advanced glycation end products. J Mol Med 2005; 83: 876-86.

55. Libby P, Plutzky J. Diabetic macrovascular disease: the glucose paradox? Circulation 2002; 106: 2760-3.

56. Rahbar S, Figarola JL. Novel inhibitors of advanced glycation endproducts. Arch Biochem Biophys 2003; 419: 63-79.

57. Bonnefont-Rousselot D. Glucose and reactive oxygen species. Curr Opin Clin Nutr Metab Care 2002; 5: 561-8.

58. Lin J, Tang Y, Kang Q, Chen A. Curcumin eliminates the inhibitory effect of advanced glycation end-products 
(AGEs) on gene expression of AGE receptor-1 in hepatic stellate cells in vitro. Lab Invest 2012; 92: 827-41.

59. Lin J, Tang Y, Kang Q, Feng Y, Chen A. Curcumin inhibits gene expression of receptor for advanced glycation end-products (RAGE) in hepatic stellate cells in vitro by elevating PPARgamma activity and attenuating oxidative stress. Br J Pharmacol 2012; 166: 2212-27.

60. Tang Y, Chen A. Curcumin protects hepatic stellate cells against leptin-induced activation in vitro by accumulating intracellular lipids. Endocrinology 2010; 151: 4168-77.

61. Zeng CH, Zeng P, Deng YH, et al. [The effects of curcumin derivative on experimental steatohepatitis]. Zhonghua Gan Zang Bing Za Zhi 2011; 19: 454-9.

62. Ejaz A, Wu D, Kwan P, Meydani M. Curcumin inhibits adipogenesis in 3T3-L1 adipocytes and angiogenesis and obesity in C57/BL mice. J Nutr 2009; 139: 919-25.

63. Hasan ST, Zingg JM, Kwan P, Noble T, Smith D, Meydani M. Curcumin modulation of high fat diet-induced atherosclerosis and steatohepatosis in LDL receptor deficient mice. Atherosclerosis 2014; 232: 40-51.

64. Friedman SL. Mechanisms of hepatic fibrogenesis. Gastroenterology 2008; 134: 1655-69.

65. Miyahara T, Schrum L, Rippe R, et al. Peroxisome proliferator-activated receptors and hepatic stellate cell activation. J Biol Chem 2000; 275: 35715-22.

66. Gruzman A, Babai G, Sasson S. Adenosine monophosphate-activated protein kinase (AMPK) as a new target for antidiabetic drugs: a review on metabolic, pharmacological and chemical considerations. Rev Diabet Stud 2009; 6: 13-36.

67. You M, Matsumoto M, Pacold CM, Cho WK, Crabb DW. The role of AMP-activated protein kinase in the action of ethanol in the liver. Gastroenterology 2004; 127: 1798-808.

68. Kang Q, Chen A. Curcumin suppresses expression of low-density lipoprotein (LDL) receptor, leading to the inhibition of LDL-induced activation of hepatic stellate cells. Br J Pharmacol 2009; 157: 1354-67.

69. Kang Q, Chen A. Curcumin eliminates oxidized LDL roles in activating hepatic stellate cells by suppressing gene expression of lectin-like oxidized LDL receptor-1. Lab Invest 2009; 89: 1275-90.

70. Kang Q, Chen A. Curcumin inhibits srebp-2 expression in activated hepatic stellate cells in vitro by reducing the activity of specificity protein-1. Endocrinology 2009; 150: 5384-94.

71. Vizzutti F, Provenzano A, Galastri S, et al. Curcumin limits the fibrogenic evolution of experimental steatohepatitis. Lab Invest 2010; 90: 104-15.

72. Pinlaor S, Prakobwong S, Hiraku Y, Pinlaor P, Laothong $\mathrm{U}$, Yongvanit P. Reduction of periductal fibrosis in liver fluke-infected hamsters after long-term curcumin treatment. Eur J Pharmacol 2010; 638: 134-41.

73. Rajagopalan R, Sridharana S, Menon VP. Hepatoprotective role of bis-demethoxy curcumin analog on the expression of matrix metalloproteinase induced by alcohol and polyunsaturated fatty acid in rats. Toxicol Mech Methods 2010; 20: 252-9.

74. Jiang Y, Li ZS, Jiang FS, Deng X, Yao CS, Nie G. Effects of different ingredients of zedoary on gene expression of HSC-T6 cells. World J Gastroenterol 2005; 11: 6780-6.

75. Marra F, Efsen E, Romanelli RG, et al. Ligands of peroxisome proliferator-activated receptor gamma modulate profibrogenic and proinflammatory actions in hepatic stellate cells. Gastroenterology 2000; 119: 466-78.

76. Galli A, Crabb D, Price D, et al. Peroxisome proliferator-activated receptor gamma transcriptional regula- tion is involved in platelet-derived growth factor-induced proliferation of human hepatic stellate cells. Hepatology 2000; 31: 101-8.

77. Salzano A, Arcopinto M, Marra AM, et al. Klinefelter syndrome, cardiovascular system, and thromboembolic disease: review of literature and clinical perspectives. Eur J Endocrinol 2016; 175: R27-40.

78. Ellulu MS, Patimah I, Khaza'ai H, Rahmat A, Abed Y. Obesity and inflammation: the linking mechanism and the complications. Arch Med Sci 2017; 13: 851-63.

79. Hajavi J, Momtazi AA, Johnston TP, Banach M, Majeed M, Sahebkar A. Curcumin: a naturally occurring modulator of adipokines in diabetes. J Cell Biochem 2017; 118: 4170-82.

80. Kim SW, Ha KC, Choi EK, et al. The effectiveness of fermented turmeric powder in subjects with elevated alanine transaminase levels: a randomised controlled study. BMC Complement Altern Med 2013; 13: 58.

81. Angulo P. Treatment of nonalcoholic fatty liver disease. Ann Hepatol 2002; 1: 12-9.

82. Vernon G, Baranova A, Younossi ZM. Systematic review: the epidemiology and natural history of non-alcoholic fatty liver disease and non-alcoholic steatohepatitis in adults. Aliment Pharmacol Ther 2011; 34: 274-85.

83. Begriche K, Igoudjil A, Pessayre D, Fromenty B. Mitochondrial dysfunction in NASH: causes, consequences and possible means to prevent it. Mitochondrion 2006; 6: 1-28.

84. Machado MV, Cortez-Pinto H. Non-alcoholic fatty liver disease: what the clinician needs to know. World J Gastroenterol 2014; 20: 12956-80.

85. Asgharian A, Askari G, Esmailzade A, Feizi A, Mohammadi $V$. The effect of symbiotic supplementation on liver enzymes, C-reactive protein and ultrasound findings in patients with non-alcoholic fatty liver disease: a clinical trial. Int J Prev Med 2016; 7: 59.

86. Pezeshki A, Safi S, Feizi A, Askari G, Karami F. The effect of green tea extract supplementation on liver enzymes in patients with nonalcoholic fatty liver disease. Int J Prev Med 2016; 7: 28.

87. Dziedzic EA, Przychodzeń S, Dąbrowski M. The effects of vitamin $D$ on severity of coronary artery atherosclerosis and lipid profile of cardiac patients. Arch Med Sci 2016; 12: 1199-206.

88. Khan N, Akhtar MS, Khan BA, de Braga VA, Reich A. Antiobesity, hypolipidemic, antioxidant and hepatoprotective effects of Achyranthes aspera seed saponins in high cholesterol fed albino rats. Arch Med Sci 2015; 11: 1261-71.

89. Bradford PG. Curcumin and obesity. BioFactors 2013; 39: 78-87.

90. Panahi Y, Khalili N, Hosseini MS, Abbasinazari M, Sahebkar A. Lipid-modifying effects of adjunctive therapy with curcuminoids-piperine combination in patients with metabolic syndrome: results of a randomized controlled trial. Complement Ther Med 2014; 22: 851-7.

91. Chuengsamarn S, Rattanamongkolgul S, Phonrat B, Tungtrongchitr R, Jirawatnotai S. Reduction of atherogenic risk in patients with type 2 diabetes by curcuminoid extract: a randomized controlled trial. J Nutr Biochem 2014; 25: 144-50.

92. Chen WJ, Cai B, Chen HT, et al. The role of ADIPOQ methylation in curcumin-administrated experimental nonalcoholic fatty liver disease. J Dig Dis 2016; 17: 829-36. 
93. Panahi Y, Kianpour P, Mohtashami R, Jafari R, Simental-Mendía LE, Sahebkar A. Curcumin lowers serum lipids and uric acid in subjects with nonalcoholic fatty liver disease: a randomized controlled trial. J Cardiovasc Pharmacol 2016; 68: 223-9

94. Rahmani S, Asgary S, Askari G, et al. Treatment of non-alcoholic fatty liver disease with curcumin: a randomized placebo-controlled trial. Phytother Res 2016; 30: 1540-8.

95. Panahi Y, Kianpour P, Mohtashami R, Jafari R, Simental-Mendia LE, Sahebkar A. Efficacy and safety of phytosomal curcumin in non-alcoholic fatty liver disease: a randomized controlled trial. Drug Res 2017; 67: 244-51.

96. Panahi Y, Hosseini MS, Khalili N, Naimi E, Majeed M, Sahebkar A. Antioxidant and anti-inflammatory effects of curcuminoid-piperine combination in subjects with metabolic syndrome: a randomized controlled trial and an updated meta-analysis. Clin Nutr 2015; 34: 1101-8.

97. Chuengsamarn S, Rattanamongkolgul S, Luechapudi porn R, Phisalaphong C, Jirawatnotai S. Curcumin extract for prevention of type 2 diabetes. Diabetes Care 2012; 35: 2121-7.

98. Cicero AFG, Colletti A, Bajraktari G, et al. Lipid lowering nutraceuticals in clinical practice: position paper from an International Lipid Expert Panel. Arch Med Sci 2017; 13: 965-1005.

99. Proietti M, Nobili A, Raparelli V, et al. Adherence to antithrombotic therapy guidelines improves mortality among elderly patients with atrial fibrillation: insights from the REPOSI study. Clin Res Cardiol 2016; 105: 912-20.

100. Bertolotti M, Franchi C, Rocchi MB, et al. Prevalence and determinants of the use of lipid-lowering agents in a population of older hospitalized patients: the findings from the REPOSI (REgistro POliterapie Societa Italiana di Medicina Interna) Study. Drugs Aging 2017; 34: 311-9.

101. Marra AM, Arcopinto M, Bobbio E, Salzano A, Sacca L, Cittadini A. An unusual case of dilated cardiomyopathy associated with partial hypopituitarism. Intern Emerg Med 2012; 7 Suppl 2: S85-7.

102. Arcopinto M, Salzano A, Bossone E, et al. Multiple hormone deficiencies in chronic heart failure. Int J Cardiol 2015; 184: 421-3.

103. Soares MP, Bach FH. Heme oxygenase-1: from biology to therapeutic potential. Trends Mol Med 2009; 15: 50-8.

104. Zhu Z, Wilson AT, Mathahs MM, et al. Heme oxygenase- 1 suppresses hepatitis $C$ virus replication and increases resistance of hepatocytes to oxidant injury. Hepatology 2008; 48: 1430-9.

105. Choi J, Lee KJ, Zheng Y, Yamaga AK, Lai MMC, Ou JH. Reactive oxygen species suppress hepatitis $C$ virus RNA replication in human hepatoma cells. Hepatology 2004; 39: 81-9.

106. Yano M, Ikeda M, Abe K, et al. Oxidative stress induces anti-hepatitis $C$ virus status via the activation of extracellular signal-regulated kinase. Hepatology 2009; 50: 678-88.

107. Kim K, Kim KH, Kim HY, Cho HK, Sakamoto N, Cheong J. Curcumin inhibits hepatitis $C$ virus replication via suppressing the Akt-SREBP-1 pathway. FEBS Lett 2010; 584: 707-12.

108. Soni KB, Lahiri M, Chackradeo P, Bhide S V, Kuttan R. Protective effect of food additives on aflatoxin-induced mutagenicity and hepatocarcinogenicity. Cancer Lett 1997; 115: 129-33.
109. Lin LI, Ke YF, Ko YC, Lin JK. Curcumin inhibits SK-Hep-1 hepatocellular carcinoma cell invasion in vitro and suppresses matrix metalloproteinase-9 secretion. Oncology 1998; 55: 349-53.

110. Chuang SE, Kuo ML, Hsu CH, et al. Curcumin-containing diet inhibits diethylnitrosamine-induced murine hepatocarcinogenesis. Carcinogenesis 2000; 21: 331-5.

111. Thapliyal R, Naresh KN, Rao KVK, Maru GB. Inhibition of nitrosodiethylamine-induced hepatocarcinogenesis by dietary turmeric in rats. Toxicol Lett 2003; 139 : 45-54.

112. Shukla Y, Arora A. Suppression of altered hepatic foci development by curcumin in Wistar rats. Nutr Cancer 2003; 45: 53-9.

113. Sreepriya M, Bali G. Chemopreventive effects of embelin and curcumin against N-nitrosodiethylamine/phenobarbital-induced hepatocarcinogenesis in Wistar rats. Fitoterapia 2005; 76: 549-55.

114. Busquets S, Carbó N, Almendro V, Quiles MT, López-Soriano FJ, Argilés JM. Curcumin, a natural product present in turmeric, decreases tumor growth but does not behave as an anticachectic compound in a rat model. Cancer Lett 2001; 167: 33-8.

115. Notarbartolo M, Poma P, Perri D, Dusonchet L, Cervello M, D'Alessandro N. Antitumor effects of curcumin, alone or in combination with cisplatin or doxorubicin, on human hepatic cancer cells. Analysis of their possible relationship to changes in NF-kB activation levels and in IAP gene expression. Cancer Lett 2005; 224: 53-65.

116. Takaba K, Hirose M, Yoshida Y, Kimura J, Ito N, Shirai T. Effects of n-tritriacontane-16,18-dione, curcumin, chlorphyllin, dihydroguaiaretic acid, tannic acid and phytic acid on the initiation stage in a rat multi-organ carcinogenesis model. Cancer Lett 1997; 113: 39-46.

117. Hirose M, Takahashi S, Ogawa K, et al. Chemoprevention of heterocyclic amine-induced carcinogenesis by phenolic compounds in rats. Cancer Lett 1999; 143: 173-8.

118. Hirose M, Takahashi S, Ogawa K, Futakuchi M, Shirai T. Phenolics: blocking agents for heterocyclic amine-induced carcinogenesis. Food Chem Toxicol 1999; 37: 985-92.

119. Tharappel JC, Lehmler HJ, Srinivasan C, Robertson LW, Spear BT, Glauert HP. Effect of antioxidant phytochemicals on the hepatic tumor promoting activity of 3,3',4,4'-tetrachlorobiphenyl (PCB-77). Food Chem Toxicol 2008; 46: 3467-74.

120. Frank N, Knauft J, Amelung F, Nair J, Wesch H, Bartsch H. No prevention of liver and kidney tumors in Long-Evans Cinnamon rats by dietary curcumin, but inhibition at other sites and of metastases. Mutat Res 2003; 523-524: 127-35.

121. Cao H, Wang Y, He X, et al. Codelivery of sorafenib and curcumin by directed self-assembled nanoparticles enhances therapeutic effect on hepatocellular carcinoma. Mol Pharm 2015; 12: 922-31.

122. Gentile I, Borgia G. A pill a day keeps HCV away. Lancet Infect Dis 2015; 15: 616-7.

123. Gentile I, Buonomo AR, Borgia G. Ombitasvir: a potent pan-genotypic inhibitor of NS5A for the treatment of hepatitis $C$ virus infection. Expert Rev Anti Infect Ther 2014; 12: 1033-43.

124. Gentile I, Buonomo AR, Zappulo E, et al. Asunaprevir, a protease inhibitor for the treatment of hepatitis $C$ infection. Ther Clin Risk Manag 2014; 10: 493-504. 
125. Gutierrez JA, Lawitz EJ, Poordad F. Interferon-free, direct-acting antiviral therapy for chronic hepatitis $C$. J Viral Hepat 2015; 22: 861-70.

126. Gentile I, Buonomo AR, Zappulo E, Coppola N, Borgia G. GS-9669: a novel non-nucleoside inhibitor of viral polymerase for the treatment of hepatitis C virus infection. Expert Rev Anti Infect Ther 2014; 12: 1179-86.

127. Gentile I, Borgia G. Ledipasvir/Sofosbuvir administration achieves very high rate of viral clearance in patients with HCV genotype 1 infection without cirrhosis, regardless of ribavirin co-administration or length of treatment. Evid Based Med 2014; 19: 223-4.

128. Gentile I, Buonomo AR, Borgia G. Dasabuvir: a non-nucleoside inhibitor of NS5B for the treatment of hepatitis C virus infection. Rev Recent Clin Trials 2014; 9 115-23. 